\title{
Maintenance of Sinus Rhythm and Recovery of Atrial Mechanical Function After Cardioversion With Bepridil or in Combination With Aprindine in Long-Lasting Persistent Atrial Fibrillation
}

\author{
Akira Fujiki, MD; Takayuki Tsuneda, MD; Masao Sakabe, MD; Keiko Nakagawa, MD; \\ Koichi Mizumaki, MD; Tadakazu Hirai, MD; Hiroshi Inoue, MD
}

\begin{abstract}
Background The aim of this study was to evaluate pharmacological cardioversion of long-lasting persistent atrial fibrillation (AF) using bepridil in terms of recovery of atrial mechanical function and maintenance of sinus rhythm. Bepridil alone or in combination with aprindine is effective for termination of persistent AF.

Methods and Results The study group comprised 38 consecutive patients ( 24 men, 58.8 \pm 9.3 years) with successful conversion of persistent AF lasting $>1$ month either pharmacologically (Group I) or electrically (Group II). Fast Fourier transform analysis of fibrillation waves was performed and fibrillation cycle length (FCL) was calculated from the peak frequency. In Group I, sinus rhythm was pharmacologically restored in 22 patients after an average 30 days (7-49 days) of bepridil administration, either alone (11) or in combination with oral aprindine (11); they were followed up while using the same drugs. In Group II, electrical conversion restored sinus rhythm in 16 patients, and they were followed up with conventional antiarrhythmic drugs other than bepridil and aprindine. After bepridil treatment FCL increased and became significantly longer in Group I than in Group II $(190 \pm 39$ vs $150 \pm 29 \mathrm{~ms}, \mathrm{p}<0.001)$. Atrial peak velocity in transmitral flow within the first week after cardioversion was greater in Group I than in Group II $(68 \pm 35$ vs $32 \pm 20 \mathrm{~cm} / \mathrm{s}, \mathrm{p}<0.05)$. By Kaplan-Meier analysis, $83 \%$ of Group I patients were free of AF recurrence at the 12-month follow-up, compared with $36 \%$ in Group II $(\mathrm{p}<0.005)$.
\end{abstract}

Conclusions In patients with long-lasting AF, pharmacological conversion with bepridil alone or in combination with aprindine recovered atrial mechanical function better and maintained sinus rhythm longer than electrical conversion. (Circ J 2004; 68: 834-839)

Key Words: Antiarrhythmic agents; Aprindine; Atrial fibrillation; Bepridil; Defibrillation

$\mathbf{P}$ harmacological termination by class I antiarrhythmic drugs is usually considered effective treatment for atrial fibrillation (AF) lasting only several days, but of little help for long-lasting AF. As AF progresses from paroxysmal to persistent, changes in the relative importance of AF mechanisms occur such as increasing substratemediated factors and decreasing triggering factors. Hence, in patients with long lasting $\mathrm{AF}$, rhythm control therapy is abandoned and rate control therapy is selected as standard therapy 1,2 However, we have previously demonstrated that oral administration of bepridil alone or in combination with aprindine restored sinus rhythm in $69 \%$ of 32 patients with long-lasting persistent $\mathrm{AF}$ ( $>3$ months), and we speculate that bepridil inhibits substrate-mediated factors and aprindine suppresses trigger-mediated factors. Bepridil hydrochloride, a diarylaminopropylamine derivative, was introduced as an anti-anginal agent. In addition to its $\mathrm{Ca}$ antagonist properties, it prolongs action potential duration

(Received May 6, 2004; revised manuscript received June 15, 2004; accepted June 22, 2004)

The Second Department of Internal Medicine, Toyama Medical and Pharmaceutical University, Toyama, Japan

Mailing address: Akira Fujiki, MD, The Second Department of Internal Medicine, Toyama Medical and Pharmaceutical University, 2630 Sugitani, Toyama 930-0194, Japan. E-mail: fujiki@ms.toyama-mpu. ac.jp and refractory periods of normal ventricular and atrial myocardium 4 by inhibiting several types of $\mathrm{K}$ current including rapid, slow and ultra rapid components of delayed rectifier K current5,6 Amiodarone has multi-channel blocking properties similar to bepridil and is effective in not only terminating long-lasting $\mathrm{AF}$, but also maintaining sinus rhythm? In a canine model of AF, amiodarone prevented down-regulation of the L-type $\mathrm{Ca}$ channel and increased fibrillation cycle length (FCL) through reversal of atrial remodeling?

In our previous study, termination of long-lasting $\mathrm{AF}$ with oral administration of bepridil was associated with a $30 \%$ increase in FCL2 and it is possible that bepridil is effective for not only termination of AF but also perpetuation of sinus rhythm. In the present study, we assessed the efficacy of pharmacological or electrical conversion in patients with long-lasting AF for recovery of atrial mechanical function and maintenance of sinus rhythm with respect to changes in FCL.

\section{Methods}

Subjects

All 38 patients in the study group had had AF lasting at least 1 month, quantified by ECG, and the median was 5 months (range: 1-84 months). All patients underwent phys- 
ical examination, 12-lead ECG, echocardiography and biochemical and hematological testing. Excluded were women of child-bearing age and patients aged over 80 years, with a myocardial infarction or revascularization within the past 3 months, left ventricular ejection fraction (LVEF) $<0.40$, QTc $>0.46 \mathrm{~s}$, serum $\mathrm{K}<3.8 \mathrm{mmol} / \mathrm{L}$ or a history of sick sinus syndrome. All patients had undergone anticoagulation therapy with warfarin $(\mathrm{INR} \fallingdotseq 2.0)$ for at least 3 weeks before either electrical or pharmacological conversion was attempted. Concurrent control of the ventricular rate with $\mathrm{Ca}$ antagonists, $\beta$-blocking drugs or digitalis was permitted.

\section{Study Protocol}

Two groups were studied: Group I consisted of 22 consecutive patients (14 men, average age $57.4 \pm 8.7$ years) with persistent AF who had sinus rhythm restored successfully by oral bepridil alone (11 patients) or in combination with aprindine (11 patients). Group II consisted of 16 consecutive patients (10 men, average age $60.9 \pm 10.3$ years) who had sinus rhythm restored electrically and were followed up with conventional antiarrhythmic drugs other than bepridil and aprindine. The mode of conversion to sinus rhythm was selected by patient preference. We informed patients of the success rate of both chemical and electrical cardioversion. Because the patients with the longer duration of AF had already adjusted their life style to rate control therapy, they tended to select chemical cardioversion rather than electrical cardioversion. The details of the Group I protocol have been published previously? Briefly, after informed consent was obtained, oral bepridil ( $200 \mathrm{mg}$ /day) was started, and patients were followed for 4 weeks. If bepridil failed to restore sinus rhythm and the QTc interval was not prolonged markedly, oral aprindine (40 or $60 \mathrm{mg} /$ day) was added and patients were followed for another 4 weeks. After pharmacological conversion, the treatment was continued at the same dosage unless marked QT prolongation or sinus bradycardia appeared. During sinus rhythm and AF, QT interval was corrected (QTc) by dividing the measured QT interval by the square root of the preceding RR interval that showed the minimal difference between the average values of RR intervals.

In Group II, after the intravenous administration of thiopental $(4 \mathrm{mg} / \mathrm{kg})$, patients were shocked with R-wave synchronized monophasic discharges (F-1300, Fukuda Denshi, Tokyo, Japan) beginning with $100 \mathrm{~J}$ and ranging up to $300 \mathrm{~J}$ until cardioversion was achieved (antero-lateral approach). Successful cardioversion was defined as sinus rhythm recovery lasting at least $1 \mathrm{~min}$ after the shock. No antiarrhythmic drug was given before cardioversion other than the rate control drugs.

\section{Frequency Analysis}

Spectral analyses of the fibrillation waves were performed before conversion to sinus rhythm in both groups as in our previous study? Surface ECG lead $V_{1}$ was digitally stored on-line on a microcomputer (Value Star NX, NEC, Tokyo, Japan), and QRST complexes were subtracted using a template-matching algorithm. ECG-segments were digitized at a sampling rate of $1 \mathrm{kHz}$. In order to assess the temporal pattern of the fibrillation waves, analyses of 44-s electrograms were performed.

Frequency analysis of the subtracted electrograms involved 3 steps: bandpass filtering, application of a Hamming window and 4096-point fast Fourier transformation. A $50 \%$ overlap of adjacent spectral analysis allowed the
Table 1 Baseline Clinical Characteristics of the Patients With Persistent Atrial Fibrillation Converted Pharmacologically (Group I) or Electrically (Group II)

\begin{tabular}{lcc}
\hline \hline & $\begin{array}{c}\text { Group I } \\
(n=22)\end{array}$ & $\begin{array}{c}\text { Group II } \\
(n=16)\end{array}$ \\
\hline Age (years) & $57.4 \pm 8.7$ & $60.9 \pm 10.3$ \\
$M / F$ & $14 / 8$ & $10 / 6$ \\
Duration of AF (months) & & \\
$\quad$ Mean & $17.8 \pm 22.6$ & $5.0 \pm 6.7 *$ \\
Median (range) & $6(3-84)$ & $2(1-24)$ \\
Underlying heart disease & & \\
Valvular disease & 8 & 4 \\
Congenital disease & 0 & 3 \\
Hypertension & 5 & 7 \\
None & 9 & $43.9 \pm 10.1$ \\
LA diameter (mm) & $44.2 \pm 8.6$ & $0.58 \pm 0.12$ \\
LVEF & $0.64 \pm 0.08$ & $0.41 \pm 0.02$ \\
$Q T c$ interval & $0.39 \pm 0.04$ & \\
\hline
\end{tabular}

Data are presented as mean value $S D$, unless otherwise indicated. AF, atrial fibrillation; $L A$, left atrium; $L V E F$, left ventricular ejection fraction; $Q T c$, corrected $Q T$ interval. ${ }^{*} p<0.05$.

use of averages of 20 epochs of analyses within a single data set. Power spectra were quantified by measuring the peak frequency signal with the maximum magnitude derived from each epoch. The peak frequency of the spectrum in the $3-12 \mathrm{~Hz}$ range was converted to a cycle length (cycle length in $\mathrm{ms}=1,000 /$ frequency), named the FCL, which was averaged from 20 epochs.

\section{Atrial Mechanical Function Analysis}

To assess atrial peak velocity in transmitral flow and the atrial filling/early filling (A/E) ratio, 2-dimensional imaging and pulsed Doppler studies from the apical 4chamber view were performed using transthoracic echocardiography (Toshiba 380A, Tokyo, Japan) with a $2.5 \mathrm{MHz}$ transducer. Recordings were obtained at the end of expiration with the patients in the supine or left lateral position. The sample volume was positioned between the tips of the mitral leaflets. Peak velocities of the early filling (E) wave and the atrial filling (A) wave were determined.

\section{Statistical Analysis}

All data are expressed as mean \pm SD. Paired and unpaired t-tests were used for comparison of the 2 groups of results. A Kaplan-Meier analysis with the log rank test was used to compare the probability of freedom from recurrence of AF. Results were considered to be statistically significant at $\mathrm{p}<0.05$. All statistical analyses were performed with the Statview for Windows program (Abacus Concepts, Inc, Berkeley, CA, USA).

\section{Results}

\section{Baseline Characteristics (Table 1)}

AF had been present longer in Group I than in Group II (17.8 \pm 22.6 vs 5.0 \pm 6.7 months, $\mathrm{p}<0.05)$. LVEF and left atrial dimension did not differ between the 2 groups (Group I vs II: $0.64 \pm 0.08$ vs $0.58 \pm 0.12$ and $44.2 \pm 8.6$ vs $43.9 \pm$ $10.1 \mathrm{~mm}$, respectively). In Group I, 13 patients $(59 \%)$ had structural heart disease, including 5 with hypertensive heart disease and 8 with valvular disease, compared with 9 patients $(56 \%)$ in Group II, including 3 with hypertensive heart disease, 4 with valvular disease, and 2 with congenital heart disease. 

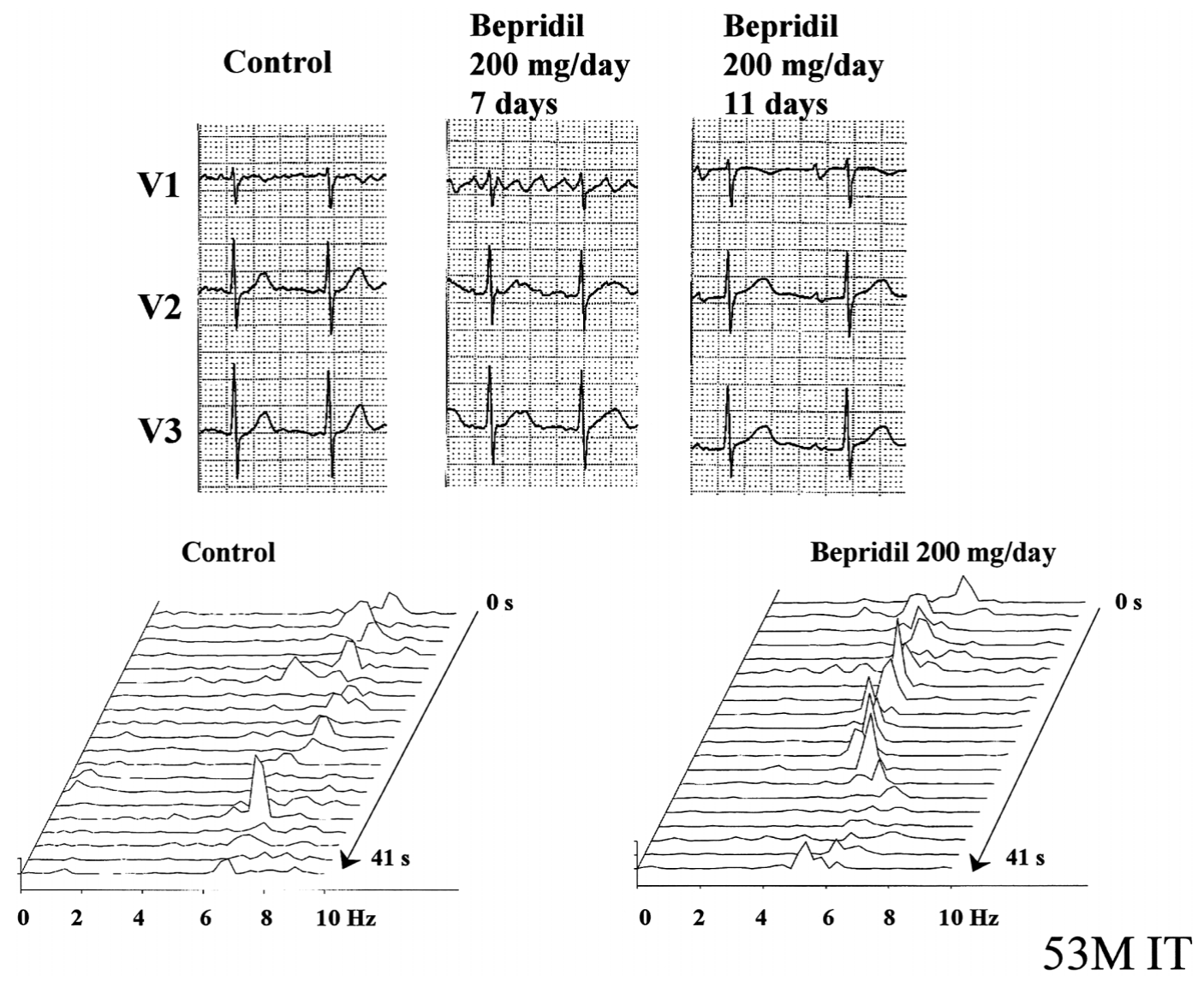

Fig 1. ECGs and spectral analyses of the fibrillation waves in a representative patient (53-year-old male) with persistent $\mathrm{AF}$ lasting 11 months that was converted by bepridil. Before bepridil administration QTc was 0.44 (QT $0.36 \mathrm{~s}$ at RR $0.68 \mathrm{~s}$ ), and fine fibrillation waves were observed in V1 (Upper left). Seven days after bepridil (200 mg/day) QTc was increased to 0.48 (QT $0.40 \mathrm{~s}$ at RR $0.68 \mathrm{~s}$ ), and fibrillation waves became coarser in $\mathrm{V}_{1}$ (Upper middle). After 11 days of bepridil, sinus rhythm was restored (Upper right). During sinus rhythm QTc was 0.43 (QT 0.40s at RR 0.88s). The peak frequency shifted to the left 7 days after bepridil therapy started. Mean FCL calculated from the peak frequency was $136 \mathrm{~ms}$ before bepridil (Lower left) and increased to $174 \mathrm{~ms}$ after bepridil therapy (Lower right).

\section{Pharmacological conversion}

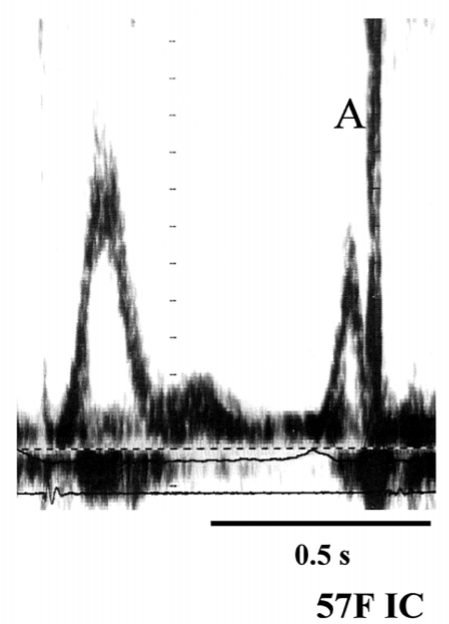

\section{Electrical conversion}

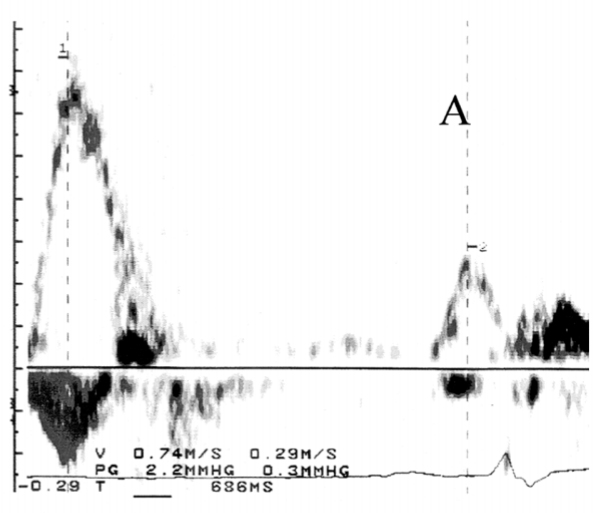

41M MY

Fig 2. Atrial mechanical function after cardioversion evaluated by pulsed-Doppler echocardiography. (Left panel) Peak velocity of the A wave was $91 \mathrm{~cm} / \mathrm{s} 4$ days after pharmacological conversion (57-year-old female without structural heart disease). (Right panel) Peak velocity of the A wave was $29 \mathrm{~cm} / \mathrm{s} 7$ days after electrical conversion (41-year-old male without structural heart disease). 


\section{Conversion to Sinus Rhythm}

In Group I, the final success rate of conversion with bepridil alone (11 patients) or in combination with aprindine (11 patients) was $69 \%$ (22 of 32 patients), and the time to conversion after starting bepridil was $30 \pm 12$ days (bepridil alone, $21 \pm 11$ days and a combination of bepridil and aprindine, $39 \pm 5$ days). Representative ECGs and spectral analyses from a patient with bepridil-induced termination are shown in Fig 1. After bepridil treatment, the FCL increased and was longer in Group I than in Group II $(190 \pm 39$ vs $150 \pm 29 \mathrm{~ms}, \mathrm{p}<0.01)$. After bepridil treatment, QTc interval increased to $0.43 \pm 0.04 \mathrm{~s}$, and the increase was $11 \pm 14 \%$. After cardioversion, the bepridil dosage was reduced from $200 \mathrm{mg}$ to $100 \mathrm{mg}$ /day in 7 patients because of QT prolongation or sinus bradycardia, but no adverse effects necessitating drug discontinuation occurred.

In Group II, successful electrical cardioversion was achieved with mean energy of $180 \pm 32 \mathrm{~J}$ (from 100 to 200 J). Antiarrhythmic drugs administered after cardioversion were sotalol in 5 patients, cibenzoline in 5, pilsicainide in 3 and amiodarone in 3.

\section{Recovery of Atrial Mechanical Function}

Representative pulsed-Doppler echocardiograms from a patient with bepridil-induced conversion and from a patient with electrical conversion are shown in Fig 2. The atrial peak velocity in transmitral flow and the $\mathrm{A} / \mathrm{E}$ ratio within the first week after cardioversion ( $4 \pm 3$ days) were greater in Group I than in Group II $(68 \pm 35$ vs $32 \pm 20 \mathrm{~cm} / \mathrm{s}, \mathrm{p}<0.05$ and $0.92 \pm 0.60$ vs $0.42 \pm 0.34, \mathrm{p}<0.05)$. The atrial peak velocity and the $\mathrm{A} / \mathrm{E}$ ratio did not change significantly between the first week and the first month after cardioversion in Group I $(68 \pm 35$ vs $77 \pm 25 \mathrm{~cm} / \mathrm{s}$ and $0.92 \pm 0.60$ vs $0.92 \pm 0.21)$.

\section{Recurrence of $A F$}

By Kaplan-Meier analysis (Fig 3), 83\% of the patients (Group I) who had responded to pharmacological conversion were free of AF recurrence at the 12-month follow-up examination, compared with $36 \%$ of the patients (Group II) who had been converted electrically and followed up with conventional antiarrhythmic drugs other than bepridil and aprindine $(\mathrm{p}<0.005)$.

\section{Discussion}

The present study demonstrated that pharmacological conversion with bepridil alone or in combination with aprindine recovered atrial mechanical function better and maintained sinus rhythm longer than electrical conversion in patients with long-lasting persistent AF. Before cardioversion, the FCL was significantly greater in the pharmacological conversion group than in the electrical converters, which may reflect reversal of atrial remodeling leading to early recovery of atrial stunning.

\section{Termination of Long-Lasting AF and Maintenance of Sinus Rhythm With Respect to FCL}

Human AF is associated with marked shortening of action potential duration and FCL as electrical remodeling progresses. ${ }^{10}$ A shortened FCL will favor transition to multiple reentry, which is a final common pathway irrespective of various initial mechanisms of AF. In the present study, pharmacological termination of long-lasting AF with bepridil alone or in combination with aprindine was pre-

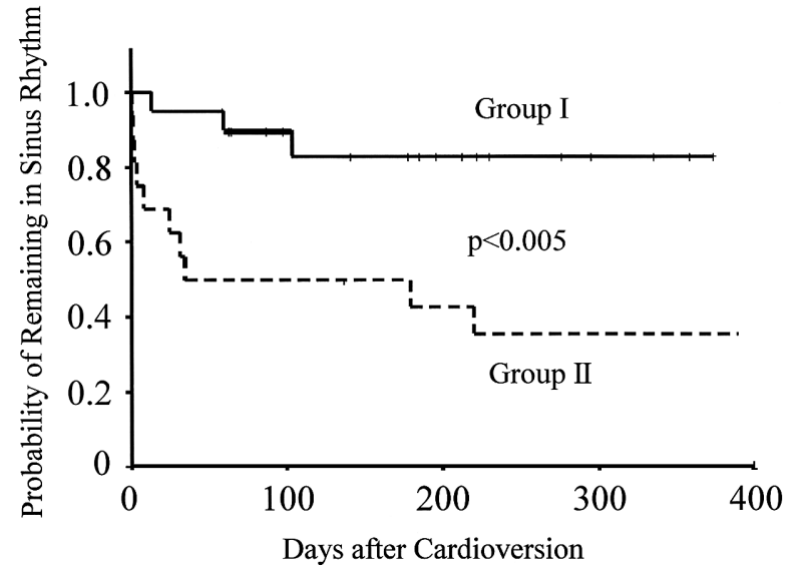

Fig 3. Kaplan-Meier curves for probability of maintaining sinus rhythm in the pharmacological and electrical conversion groups. At the 12-month follow-up examination, $83 \%$ of the patients who had been converted to sinus rhythm pharmacologically (Group I) were free of AF recurrence, compared with $36 \%$ of the patients who had been converted electrically (Group II).

ceded by a greater increase in FCL $(190 \pm 39 \mathrm{~ms})$ compared with electrical termination $(150 \pm 29 \mathrm{~ms})$. Although the gradual increase in FCL and long pharmacological conversion interval ( $30 \pm 12$ days) suggest that termination of $\mathrm{AF}$ by bepridil may be related to reversal of atrial remodeling, its precise mechanism is still unclear.

In a canine rapid pacing-induced AF model, amiodarone prevented a reduction in the expression of the L-type $\mathrm{Ca}$ channel subunit protein and pacing-induced changes in atrial refractoriness associated with prolongation of FCL? Amiodarone effects spontaneous termination of persistent $\mathrm{AF}$ and increases the efficacy of electrical cardioversion because it may reverse already established remodeling? The time-dependent reversal of remodeling by amiodarone and bepridil may be related to delayed pharmacological conversion. The increase in FCL associated with bepridil is in some part attributable to direct effects on several $\mathrm{K}$ channels, but the time course of the action of bepridil suggests an additional effect on channel protein expression.

In the present study sinus rhythm was maintained better in patients with pharmacological cardioversion by bepridil than in patients with electrical cardioversion. During pharmacological cardioversion bepridil may reverse atrial remodeling gradually and finally terminate AF. On the other hand, electrical cardioversion terminates AF rapidly with persistence of the remodeled AF substrate. These differences in the atrial substrate after termination of AF may contribute to the lower recurrence rate of $\mathrm{AF}$ in patients with pharmacological cardioversion by bepridil than with electrical cardioversion. Hence, the present study revealed the superiority of patients who had the atrial substrate converted to sinus rhythm by bepridil for maintenance of sinus rhythm but not bepridil therapy per se.

\section{Recovery of Atrial Mechanical Function}

Left atrial mechanical dysfunction after cardioversion is implicated in the development of thromboembolic stroke and the relationship between atrial mechanical stunning and the mode of cardioversion, whether electrical or pharmacological ${ }^{11}$ is unclear, because in most studies pharmacological cardioversion has been attempted by intravenous administration of class I antiarrhythmic drugs. 
In the present study, oral administration of bepridil terminated long-lasting $\mathrm{AF}$ after approximately 1 month and after pharmacological cardioversion, atrial mechanical function recovered faster and did not change between the first week and the first month after cardioversion, which suggests that pharmacological conversion by bepridil may prevent the occurrence of thromboembolic events.

Post-cardioversion atrial stunning in humans seems to be the result of a functional loss of contraction caused by reduction of the L-type Ca current.$^{12}$ Findings from a canine model of chronic rapid atrial pacing suggested that not only reduced systolic $\mathrm{Ca}$ transient but also impaired cellular $\mathrm{Ca}$ handling may contribute to atrial contractile dysfunction caused by sustained atrial tachycardia ${ }^{13}$ Electrical remodeling is reversible within a few days after restoration of sinus rhythm, whereas recovery from mechanical stunning requires a longer time, depending on the duration of $\mathrm{AF}$. Manning et al reported that recovery was achieved within 1 week in patients with moderate duration of AF (2-6 weeks) and within 1 month in patients with longer duration $(>6$ weeks) $!^{4}$ Nishino et al demonstrated that atrial mechanical function showed no change over the first week and improved between 1 and 4 weeks after conversion in patients with longer duration of AF ( $>6$ months) ${ }^{15}$ Those reports suggest that recovery of atrial mechanical function in Group I (the median duration of $\mathrm{AF}$ was 6 months) requires more than 1 month.

In the present study, pharmacological cardioversion with bepridil or in combination with aprindine significantly enhanced the recovery of atrial mechanical function. Both bepridil and amiodarone have a T-type Ca channel blocking action similar to mibefradil, which attenuates atrial tachycardia-induced electrical remodeling, ${ }^{16,17}$ and may play a role in recovery of not only electrical remodeling but also post-cardioversion atrial stunning. In cultured rat ventricular myocytes, bepridil increased the $\mathrm{Ca}$ sensitivity of contractile proteins and offset the negative inotropic effect of the L-type Ca channel blocking action! ${ }^{18}$ This $\mathrm{Ca}$ sensitizing effect may also contribute to the early recovery of atrial mechanical function.

\section{Safety of Bepridil}

Bepridil is a multi-channel blocker inhibiting both Land T-type $\mathrm{Ca}$ currents as well as $\mathrm{Na}$ current in isolated cardiac myocytes! ${ }^{17}$ Bepridil also inhibits several K currents including the ultra-rapid, rapid and slow components of the delayed rectifier K current 5,6 Muscarinic acetylcholine receptor-operated K current ${ }^{19}$ and ATP-sensitive K current ${ }^{20}$ However, these actions may contribute to marked prolongation of QT interval and QT dispersion, and possible risk for torsades de pointes.21 Perelman et al reported that oral bepridil (200-600 mg/day) was associated with development of serious ventricular arrhythmias because of excessive prolongation of QT interval?2 In the present study, however, the maximum dosage of bepridil was $200 \mathrm{mg} /$ day, and serum $\mathrm{K} \geq 3.8 \mathrm{mmol} / \mathrm{L}$ was maintained to avoid its proarrhythmic effects.

\section{Study Limitations}

First, the present study was not randomized because conversion to sinus rhythm by bepridil is possible for patients with AF lasting up to 7 years who are usually not indicated for electrical cardioversion. Hence, in the present study the mode of conversion was selected by patient preference after informed consent. Because of the non-randomized study design, the duration of $\mathrm{AF}$ tended to be longer in Group I than in Group II; however, Group I had better maintenance of sinus rhythm than Group II. Second, patients with preserved left ventricular ejection fractions $>0.40$ were included in the present study, but AF in patients with congestive heart failure might respond differently to bepridil. Further studies are needed to clarify the clinical efficacy and safety of bepridil in patients with left ventricular dysfunction. Third we selected aprindine as the additional drug because of its unique electrophysiological properties including suppression of inactivated $\mathrm{Na}$ channels ${ }^{2}{ }^{3}$ delayed rectifier $\mathrm{K}$ currents, muscarinic acetylcholine receptor-operated $\mathrm{K}$ current and hyperpolarization-activated inward current ${ }^{24}$ but it is possible that similar results could be obtained by other class I antiarrhythmic drugs. Finally, the present study demonstrated the superiority of conversion of the atrial substrate by bepridil for sinus maintenance but not of bepridil therapy per se. It is possible that the patients who responded to bepridil therapy may have shown good atrial mechanical recovery and maintenance of sinus rhythm even after electrical cardioversion followed with conventional antiarrhythmic drugs. If bepridil had been given alone or in combination with aprindine in Group II, the follow-up data might have been different and further studies, including randomization, are needed to clarify these points. Although limited for these reasons, the present study showed that pharmacological conversion by bepridil alone or in combination with aprindine might improve recovery of atrial mechanical function and longer maintenance of sinus rhythm in patients with long-lasting AF.

\section{Acknowledgments}

We gratefully acknowledge the technical assistance of Toshiaki Shimizu, BS, and Shunichi Miyamoto, BS, from Hokuriku-Fukuda Denshi.

\section{References}

1. Van Gelder IC, Hagens VE, Bosker HA, Kingma JH, Kamp O, Kingma T, et al. A comparison of rate control and rhythm control in patients with recurrent persistent atrial fibrillation. $N$ Engl $J$ Med 2002; 347: 1834-1840.

2. Yamashita T, Ogawa S, Aizawa Y, Atarashi H, Inoue H, Ohe T, et al. Investigation of the optimal treatment strategy for atrial fibrillation in Japan. Circ J 2003; 67: 738-741.

3. Fujiki A, Tsuneda T, Sugao M, Mizumaki K, Inoue H. Usefulness and safety of bepridil in converting persistent atrial fibrillation to sinus rhythm. Am J Cardiol 2003; 92: 472-475.

4. Kato R, Singh BN. Effects of bepridil on the electrophysiologic properties of isolated canine and rabbit myocardial fibers. Am Heart J 1986; 111: 271-279.

5. Wang JC, Kiyose T, Kiriyama K, Arita M. Bepridil differentially inhibits two delayed rectifier $\mathrm{K}$ currents, Ikr and Iks, in guinea-pig ventricular myocytes. Br J Pharmacol 1999; 128: 1733-1738.

6. Kobayashi S, Reien Y, Ogura T, Saito T, Masuda Y, Nakaya H. Inhibitory effect of bepridil on hKv1.5 channel current: Comparison with amiodarone and E-4031. Eur J Pharmacol 2001; 430: 149157.

7. Deedwania PC, Singh BN, Ellenbogen K, Fisher S, Flestcher R, Singh SN. Spontaneous conversion and maintenance of sinus rhythm by amiodarone in patients with heart failure and atrial fibrillation: Observations from the veterans affairs congestive heart failure survival trial of antiarrhythmic therapy (CHF-STAT). Circulation 1998; 98: $2574-2579$.

8. Shinagawa K, Shiroshita-Takeshita A, Schram G, Nattel S. Effects of antiarrhythmic drugs on fibrillation in the remodeled atrium: Insights into the mechanism of the superior efficacy of amiodarone. Circulation 2003; 107: 1440-1446.

9. Fujiki A, Sakabe M, Nishida K, Mizumaki K, Inoue H. Role of fibrillation cycle length in spontaneous and drug-induced termination of human atrial fibrillation. Circ J 2003; 67: 391-395.

10. Niwano S, Wakisaka Y, Kojima J, Yumoto Y, Inuo K, Hara H, et al. Monitoring the progression of the atrial electrical remodeling in pa- 
tients with paroxysmal atrial fibrillation. Circ J 2003; 67: 133-138.

11. Falk RH, Decara J, Abascal V. Is pharmacologic cardioversion of atrial fibrillation really preferable to electrical cardioversion? $\mathrm{J} \mathrm{Am}$ Coll Cardiol 1998; 31: 1446-1447.

12. Schotten U, Ausma J, Stellbrink C, Sabatschus I, Miriam V, Dirk F, et al. Cellular mechanisms of depressed atrial contractility in patients with chronic atrial fibrillation. Circulation 2001; 103: 691-698.

13. Sun H, Gaspo R, Leblanc N, Nattel S. Cellular mechanisms of atrial contractile dysfunction caused by sustained atrial tachycardia. Circulation 1998; 98: 719-727.

14. Manning WJ, Silverman DI, Katz SE, Riley MF, Come PC, Doherty $\mathrm{RM}$, et al. Impaired left atrial mechanical function after cardioversion: Relation to the duration of atrial fibrillation. J Am Coll Cardiol 1994; 23: 1535-1540.

15. Nishino M, Hoshida S, Tanouchi J, Ito T, Kato J, Iwai K, et al. Time to recover from atrial hormonal, mechanical, and electrical dysfunction after successful electrical cardioversion of persistent atrial fibrillation. Am J Cardiol 2000; 85: 1451-1454.

16. Fareh S, Benardeau A, Thibault B, Nattel S. The T-type Ca channel blocker mibefradil prevents the development of a substrate for atrial fibrillation by tachycardia-induced atrial remodeling in dogs. Circulation 1999; 100: 2191-2197.

17. Cohen CJ, Spires S, Van Skiver D. Block of T-type Ca channels in guinea pig atrial cells by antiarrhythmic agents and $\mathrm{Ca}$ channel antagonists. J Gen Physiol 1992; 100: 703-728.
18. Ozaki H, Zaizen H, Kiyose T, Arita M. Effects of bepridil on intracellular calcium concentration and contraction in cultured rat ventricular myocytes. J Cardiovasc Pharmacol 1999; 33: 492-499.

19. Hara Y, Nakaya H. SD-3212, a new class I and IV antiarrhythmic drug: A potent inhibitor of the muscarinic acetylcholine-receptoroperated potassium current in guinea-pig atrial cells. Br J Pharmacol 1995; 116: 2750-2756.

20. Li Y, Sato T, Arita M. Bepridil blunts the shortening of action potential duration caused by metabolic inhibition via blockade of ATPsensitive $\mathrm{K}$ channels and Na-activated $\mathrm{K}$ channels. J Pharmacol Exp Ther 1999; 291: 562-568.

21. Yoshiga Y, Shimizu A, Yamagata T, Hayano T, Ueyama T, Ohmura $\mathrm{M}$, et al. Beta-blocker decreases the increase in QT dispersion and transmural dispersion of repolarization induced by bepridil. Circ J 2002; 66: $1024-1028$.

22. Perelman MS, McKenna WJ, Rowland E, Krikler DM. A comparison of bepridil with amiodarone in the treatment of established atrial fibrillation. Br Heart J 1987; 58: 339-344.

23. Kodama I, Ogawa S, Inoue H, Kasanuki H, Kato T, Mitamura H, et al. Profile of aprindine, cibenzoline, pilsicainide and pirmenol in the framework of the Sicilian Gambit. Jpn Circ J 1999; 63: 1-12.

24. Ohtomo-Sekine Y, Uemura H, Tamagawa M, Nakaya H. Inhibitory effects of aprindine on the delayed rectifier $\mathrm{K}$ current and the muscarinic acetylcholine receptor-operated $\mathrm{K}$ current in guinea-pig atrial cells. Br J Pharmacol 1999; 126: 751 -761. 